УДК 622.276

\title{
ОЦЕНКА ИНФОРМАТИВНОСТИ ОПРЕДЕЛЕНИЯ ФИЛЬТРАЦИОННЫХ ПАРАМЕТРОВ ПЛАСТА НА ОСНОВЕ ИНТЕРПРЕТАЦИИ КРИВЫХ СТАБИЛИЗАЦИИ ДАВЛЕНИЯ
}

\author{
Мартюшев Дмитрий Александрович', \\ martyushevd@inbox.ru \\ Слушкина Анна Юрьевна², \\ slushkina2@yandex.ru \\ 1 Пермский национальный исследовательский политехнический университет, \\ Россия, 614990, г. Пермь, пр. Комсомольский, 29. \\ 2 Филиал ООО «ЛУКОЙЛ-Инжиниринг» «ПермНИПИнефть» в г. Перми, \\ Россия, 614000, г. Пермь, ул. Советской Армии, 29.
}

\begin{abstract}
Актуальность работы обусловлена тем, что основным источником информации о фильтрационных параметрах пласта являются гидродинамические исследования сКважин (КВД или КВУ). Для определения достоверных параметров удаленной от сКважины зоны пласта длительность регистрации КВД и КВУ должна быть достаточно продолжительной, что приводит К потерям в добыче нефти. Для определения фильтрационных характеристик пласта, а также снижения потерь при проведении гидродинамических исследований можно использовать метод исследования скважин без их остановки - метод стабилизации давления (КСД). Однако недостаточно изученным остается вопрос анализа и обработки результатов замеров с записью кривых стабилизации давления, которые, как и КВД, могут дать требуемую информацию о параметрах пласта.

Цель: сравнение результатов определения параметров пласта на основе анализа и интерпретации кривых восстановления давления, кривых стабилизации давления и анализа добычи в программном продукте KAPPA Workstation v.5.20.01 (модули Saphir и Тораz).

Объект: терригенные коллектора месторождений Пермского края.

Методы: современный программный продукт 5-го поколения KAPPA Workstation v.5.20.01 (модули Saphir и Topaz).

Результаты. Рассматривается особо актуальная проблема интерпретации данных ГДИ в низкопроницаемых коллекторах, поскольку по причине низких фильтрационно-емкостных свойств коллектора давление в скважинах восстанавливается в течение длительных периодов времени, и, несмотря на это, полного восстановления давления добиться удается не всегда. В случаях, когда КВД являются недовосстановленными, достоверно определить фильтрационные параметры пласта невозможно, либо возможно их определить оценочно. В свою очередь, исследования методом стабилизации давления и анализ добычи в большинстве случаев имеют большую продолжительность по времени, чем исследования методом КВД, и, следовательно, на диггностическом графике можно четко выделить участок радиальной фильтрации и получать достоверные параметры пласта. В итоге проведенного анализа можно сделать вывод, что при сопоставлении результатов, полученных при интерпретации КВД, с данными при обработке кривых стабилизации давления и анализа добычи выявлена высокая сходимость и погрешность между значениями составляет менее $5 \%$.
\end{abstract}

\section{Ключевые слова:}

Гидродинамические исследования скважин, кривая стабилизации давления, кривая восстановления давления, анализ добычи, низкопроницаемые коллектора, фильтрационные параметры пласта, программный продукт КАРРА.

\section{Введение}

На сегодняшний момент основным источником информации о фильтрационных параметрах пласта являются гидродинамические исследования скважин (КВД или КВУ). Для определения достоверных параметров удаленной от скважины зоны пласта длительность регистрации КВД или КВУ должна быть достаточно продолжительной, что приводит к потерям в добыче нефти [1-4]. Все чаще интерпретация данных гидродинамических исследований (ГДИ) многими нефтедобывающими предприятиями производится в программном продукте KAPPA (модуль Saphir). Как правило, на практике исследования методом КВД в низкопроницаемых (в работе рассмотрен класс коллектора по проницаемости - низкий и средний, классификация А.А. Ханина) коллекторах и последующая обработка в программном продукте KAPPA (модуль Saphir) чаще всего малоинформативны из-за невозможности выделения радиального участка фильтрации (необходима длительная остановка скважин) и, как следствие, параметры пласта определяются неверно (оценочно) [5-14].

В настоящее время на нефтяных месторождениях все чаще глубинные насосы начинают оснащать датчиками давления (термоманометрическими системами (ТMC) или глубинными манометрами) в большинстве случаев для контроля работы насосов [15-19]. Запись изменения давления осуществляется в процессе всего времени эксплуатации скважины. На данный момент анализ данных давления, записанных с помощью глубинных датчиков, показал возможность их автоматизированной интерпретации в программном продукте КАРPA. Таким образом, для определения фильтрационных характеристик пласта, а также снижения потерь нефти при проведении гидродинамических исследований появилась возможность широко использовать метод исследования скважин без их остановки - метод стабилизации давления 
(КСД). Однако недостаточно изученным остается вопрос анализа и обработки результатов замеров с записью кривых стабилизации давления, которые, как и КВД, могут дать требуемую информацию о параметрах пласта.

Еще одним инструментом, который может служить для определения параметров пласта, является метод анализа добычи (АД), который реализован в программном комплексе KAPPA Workstation v5.20.01 модуль Topaz. Стоить отметить, что для получения качественных данных необходимы данные длительного мониторинга работы скважины - в течение нескольких месяцев, желательно с момента ввода ее в эксплуатацию [19-22]. Преимуществом обработки данных в Topaz является то, что получение фильтрационно-емкостных параметров пласта не требует дополнительных исследований и, как следствие, сокращаются потери добычи и не требуется дополнительных экономических вложений.

Исследования методом КСД и АД в отличие от КВД имеют большую продолжительность исследования и на диагностическом графике можно однозначно наблюдать выделение прямолинейного участка, характеризующего радиальный фильтрационный поток, и, соответственно, определенные параметры пласта будут являться достоверными.

На сегодняшний момент на месторождениях Пермского края по результатам оценки около 45-50\% гидродинамических исследований (КВД или КВУ) пластовое давление и фильтрационноеемкостные характеристики пласта определены оценочно. Таким образом, целью данной работы является сравнение достоверности определения характеристик пласта (проницаемость и скин-фактор) на основе анализа и интерпретации кривых стабилизации давления (КСД), кривых восстановления давления (КВД) и анализа добычи (АД) в программном продукте KAPPA Workstation v5.20.01 (модули Saphir и Topaz).

\section{Интерпретация данных КВД и КСД (модуль Saphir)}

Для обработки и дальнейшей интерпретации данных исследований проведен анализ истории записи давлений, по каждой скважине оценивалась следующая информация: наличие КСД; кондиционность записи; проведение исследований; отсутствие ГТМ.

Одним из основных условий, обеспечивающих качественное проведение исследований методом КСД и корректную интерпретацию, является требование по длительности отработки скважины на режиме перед остановкой на исследование. Рекомендуемое время отработки скважины на режиме должно превышать в 1,5-3 раза время начала участка радиального притока $[23,24]$. На результаты исследований существенно влияют такие характеристики, как дебит и продолжительность (необходимо учитывать всю предысторию скважины) [25]. Измерения давления в обязательном порядке должны сопровождаться данными о пред- ыстории эксплуатации скважины. Основные геолого-физические характеристики объекта Тл-Бб Шагиртско-Гожанского месторождения следующие: средняя глубина залегания пласта 1360,0 м; нефтенасыщенная толщина пласта 3,5-5,7 м; начальное пластовое давление 14,6 МПа; пористость - $19 \%$; проницаемость 0,22-0,59 мкм² ; вязкость нефти в пластовых условиях - 36,0 мПа.с; газосодержание - 14,2 $\mathrm{m}^{3} / \mathrm{T}$; объемный коэффициент нефти - 1,027. На рис. 1 представлена запись забойного давления и дебита жидкости по скважине № 287 Шагиртско-Гожанского месторождения Пермского края. Из анализа истории записи давления и дебита по скважине № 287 выделяется - два участка КСД и два участка проведения ГДИ, также весь временной промежуток можно использовать для анализа добычи в модуле Тораz.

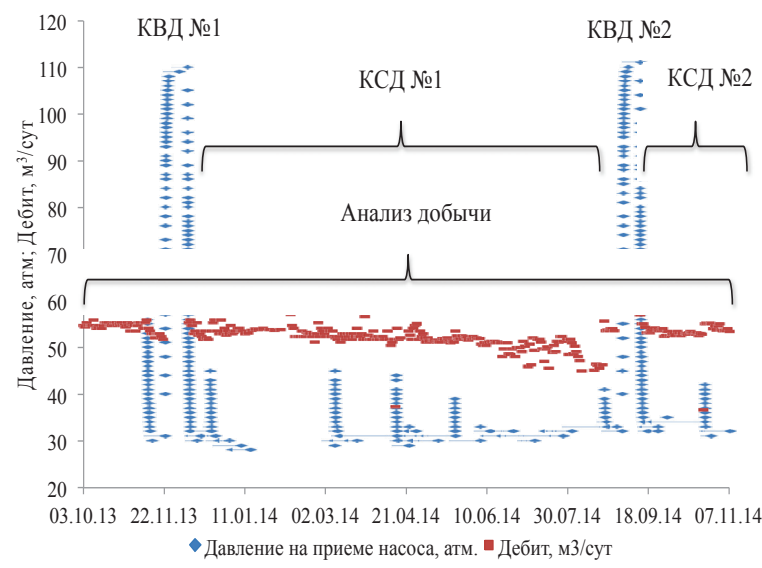

Puc. 1. Запись забойного давления и дебита жидкости. Скважи на № 287 Шагиртско-Гожанского месторождения

Fig. 1. Record of the bottomhole pressure and fluid flow rate. Well 287 of Shagirtsk-Gozhansky field

Стоить отметить, что не все исследования, полученные с ТМС или глубинных манометров, могут быть в дальнейшем интерпретированы в связи с неудовлетворительным качеством исходных данных по замерам дебитов, которые могут быть вызваны следующими причинами: отсутствие замеров дебита вовремя КСД и низкая дискретность замеров дебита. Также по ряду скважин получены некондиционные записи давлений, которые не подлежат обработке по нескольким причинам: нестабильная работа насосного оборудования и низкая разрешающая способность глубинных манометров.

Низкая разрешающая способность датчиков TMC играет значительную роль в кондиционности записи давления и в дальнейшей корректной интерпретации данных. Обычно низкая разрешающая способность датчиков не позволяет зафиксировать минимальные изменения давления, что особенно важно, когда кривая выходит на асимптоту, которая характеризует фильтрационные параметры пласта. В этих случаях зачастую провести корректную обработку данных исследований 
не предоставляется возможным. При регистрации КВД чувствительность по давлению должна быть не ниже 0,01 атм., когда для регистрации КСД может быть более 0,01 атм.

На примере скважины № 287 (рис. 1) будет рассмотрена интерпретация данных КСД, КВД и АД и произведено сопоставление полученных результатов о параметрах пласта. В модуле Saphir произведена обработка КВД № 1, диагностический график модели кривой представлен на рис. 2 (зеленыл точками показан график исходной КВД; красньми точкали - график производной КВД; красной и черной кривой представлены математические модели, описывающие график исходной и производной КВД), и совмещенная обработка КСД № 1 и 2, диагностический график модели кривой представлен на рис. 3.

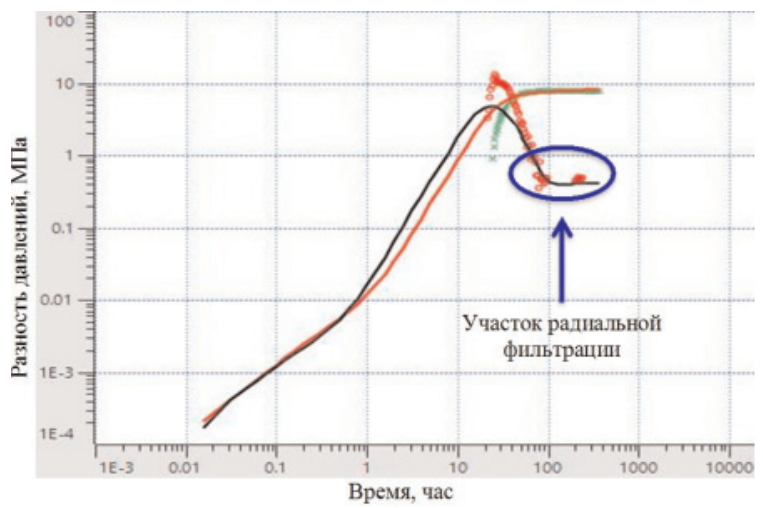

Рис. 2. Диагностический график математической модели кри вой (КВД № 1) в билогарифмических координатах

Fig. 2. Diagnostic graph of the curve mathematical model (curve no. 1 of pressure recovery) in the log-log coordinates

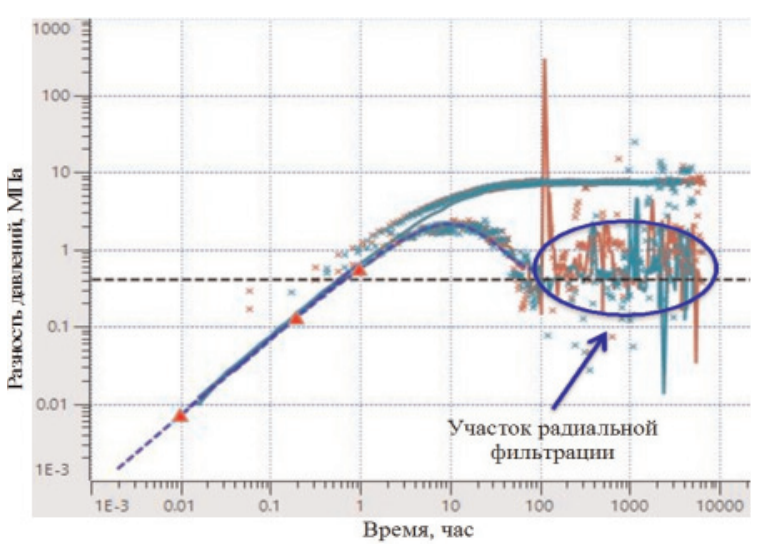

Рис. 3. Диагностический график математической модели кривой (КСД № 1 и 2 ) в билогарифмических координатах

Fig. 3. Diagnostic graph of the curve mathematical model (curve of pressure stabilization no. 1 and 2 ) in log-log coordinates

Для интерпретации КВД и КСД выбраны идентичные модели: пласт однородный бесконечный, скважина - вертикальная. Из представленных графиков можно видеть, что на диагностическом графике КВД (рис. 2) и КСД (рис. 3) четко выделяется участок радиальной фильтрации, что может гово- рить о том, что полученные параметры пласта будут являться достоверными и их сравнение можно считать корректным. Проницаемость удаленной зоны пласта и скин-фактор, полученные при обработки данным КВД и КСД, представлены в таблице.

таблица. Сравнение результатов интерпретации данных КВД, КСД и АД по скв. 287

Table. Comparison of the results of data interpretation of pressure build-up curve, pressure stabilization curves and production analysis along well 287

\begin{tabular}{|c|c|c|c|c|c|}
\hline \multirow[t]{3}{*}{$\begin{array}{c}\text { Дата исследова- } \\
\text { ния Study date }\end{array}$} & \multirow[t]{3}{*}{$\begin{array}{l}\text { Вид исследования } \\
\text { Kind of research }\end{array}$} & \multicolumn{2}{|c|}{\begin{tabular}{|c|} 
Проница- \\
емость, мд \\
Permeability, \\
mD
\end{tabular}} & \multicolumn{2}{|c|}{$\begin{array}{l}\text { Скин-фактор } \\
\text { Skin factor }\end{array}$} \\
\hline & & \multicolumn{4}{|c|}{ модуль/module } \\
\hline & & Saphir & Topaz & Saphir & Topaz \\
\hline 22.11 .2013 & $\begin{array}{c}\text { КВД № } 1 \\
\text { pressure build-up } \\
\text { curve no. } 1 \\
\end{array}$ & 13,0 & \multirow{4}{*}{17,4} & 0,54 & \multirow{4}{*}{-1} \\
\hline $08.12 .13-25.08 .14$ & $\begin{array}{c}\text { КСД № } 1 \\
\text { pressure stabiliza- } \\
\text { tion curve no. } 1\end{array}$ & 14,0 & & $-3,44$ & \\
\hline 01.09 .2014 & $\begin{array}{c}\text { КВД № } 2 \\
\text { pressure build-up } \\
\text { curve no. } 2\end{array}$ & 14,2 & & 1,53 & \\
\hline $19.09 .14-05.04 .15$ & $\begin{array}{c}\text { КСД № } 2 \\
\text { pressure stabiliza- } \\
\text { tion curve no. } 2\end{array}$ & 14,0 & & $-3,44$ & \\
\hline
\end{tabular}

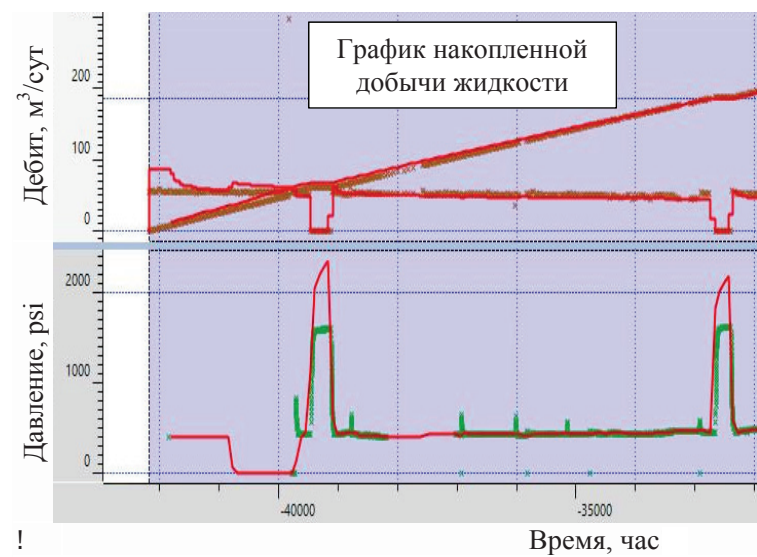

Pис. 4. График моделирования забойного давления, дебита жидкости и накопленной добычи жидкости по скважине № 287

Fig. 4. Graph of modeling the bottomhole pressure, fluid flow rate and cumulative fluid production through the well 287

\section{Интерпретация данных добычи (модуль Topaz)}

Метод анализа добычи, реализованный в программном комплексе KAPPA Workstation v5.20.01 модуль Тораz, позволяет оценить тот же набор параметров пласта, что и в модуле Saphir. Адаптация модели производится по накопленной добыче и забойному давлению, для этого строятся зависимости забойного давления, дебита жидкости и накопленной добычи от времени (рис. 4). Для получения качественных данных необходимы данные длительного мониторинга работы скважины - в течение нескольких месяцев, желательно с момента 
ввода ее в эксплуатацию. На рис. 5 представлен диагностический график математической модели кривой (коричневыл и зеленыл иветали показаны дебит и давление соответственно, залеренные с помощью ТМС; красныли кривыли показаны мателатические модели, описывающие график дебита, накопленной добычи жидкости и давления), выделенной на рис. 4. Результаты обработки данных АД представлены в таблице.

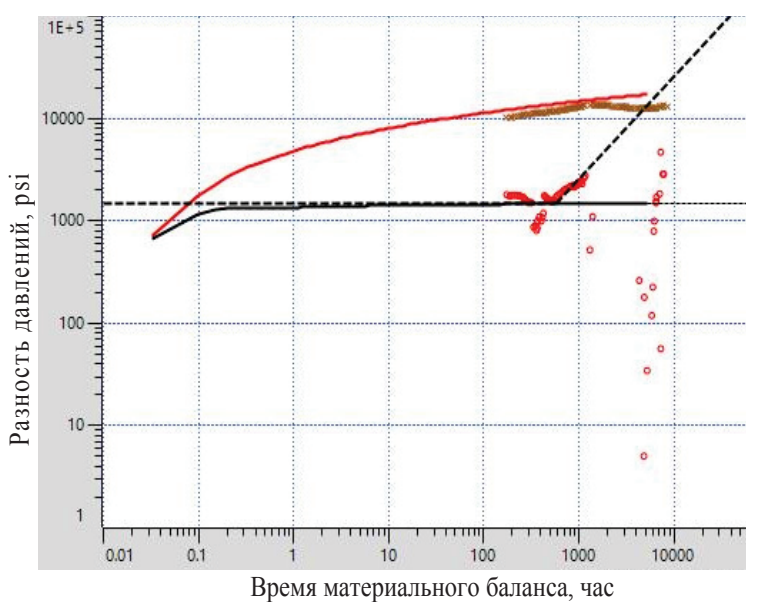

Pис.5. Диагностический график математической модели кри вой в билогарифмических координатах

Fig. 5. Diagnostic graph of the curve mathematical model in the log $\log$ coordinates

\section{Анализ полученных данных}

В таблице представлено сравнение полученных значений проницаемости и скин-фактора в результате обработки двух КСД, двух КВД и истории добычи по скважине № 287. Из данных, представленных в таблице, видно, что проницаемость пласта, определенная по результатам интерпретации КВД (КВД восстановлены на 97-99 \% ) и при обработке кривых стабилизации давления (участок № 1 и 2) находится в пределах одних числовых значений, 13 и 14 мД соответственно. При сопоставлении значений проницаемости, полученных при обработке КВД, КСД и АД расхождение между ними является незначительным. Полученные данные указывают на достоверность получаемых параметров пласта и перспективность использования методов КСД и АД на практике для оценки проницаемости и состояния призабойной зоны пласта (скин-фактора). По результатам обработки кондиционных данных записей давления и дебитов по 14 скважинам построена зависимость, представленная на рис. 6.

Все КВД, по которым построена зависимость, представленная на рис. 6, характеризовались как практически полностью восстановленными (явно выделяется участок радиальной фильтрации), то есть определенные параметры пласта можно считать корректными. В результате сравнения полу- ченных коэффициентов проницаемости установлено, что большая часть значений характеризуется высокой степенью сходимости, отклонения значений находятся в пределах $5 \%$, что может свидетельствовать о достоверности определения параметров пласта методом КСД и АД.

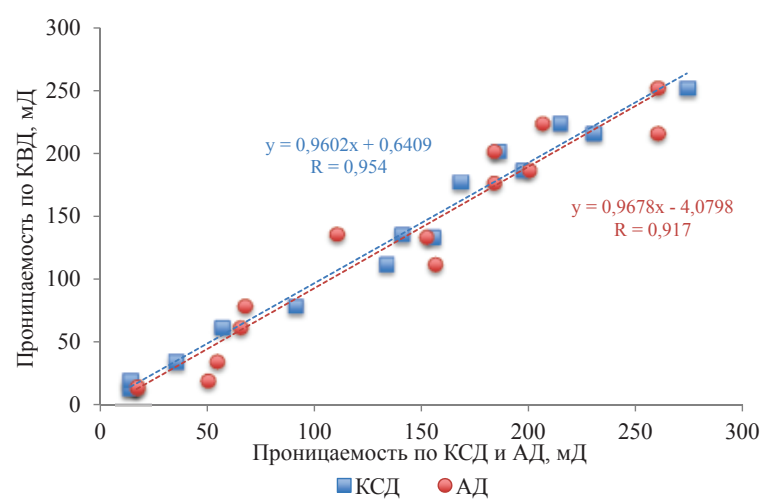

Pис.6. Зависилость проницаемости, полученной при обработке данных КВД, от пронииаелости, полученной при интерпретации данных КСД и АД

Fig. 6. Dependence of permeability obtained by processing the data of the pressure recovery curves on the permeability obtained in interpreting the data of pressure stabilization curves and production analysis

\section{Заключение}

В работе обозначена особо актуальная проблема - интерпретация данных ГДИ в низкопроницаемых коллекторах, поскольку по причине низких фильтрационно-емкостных свойств коллектора давление в скважинах восстанавливается в течение длительных периодов времени, и, несмотря на это, полного восстановления давления добиться удается не всегда. В случаях, когда КВД являются недовосстановленными, достоверно определить фильтрационные параметры пласта невозможно, либо возможно их определить лишь оценочно. В свою очередь, исследования методом стабилизации давления и анализ добычи в большинстве случаев имеют большую продолжительность по времени, чем исследования методов КВД, и, следовательно, на диагностическом графике можно четко выделить участок радиальной фильтрации и получать достоверные параметры пласта. В итоге можно сделать вывод, что при сопоставлении результатов, полученных при интерпретации КВД, с параметрами, полученными при обработке КСД и АД, получена высокая сходимость и погрешность находится в пределах $5 \%$. Таким образом, методы КСД и АД возможно использовать при определении параметров низкопроницаемых коллекторов, когда это невозможно сделать по КВД ввиду низкого качества данных (недовосстановленные КВД), и на основании информации о пласте возможно уточнение гидродинамической модели и оптимизация режимов работы скважин. 


\section{СПИСОК ЛИТЕРАТУРЫ}

1. Пономарева И.Н., Мильчаков С.В. Оптимизация периода восстановления давления в нефтедобывающих скважинах при их исследовании // Геология, геофизика и разработка нефтяных и газовых месторождений. - 2009. - № 9-10. - С. 36-39.

2. Lin Jiaen, Yang Huizhu. Pressure buildup analysis using type curves for a well in a pressure-maintained system / Chinese journal of chemical engineering. - 2007. - V. 15. - Iss. 1. - P. 6-11.

3. Управление разработкой на основе долговременного гидродинамического мониторинга на примере западно-салымского месторождения / Д.Н. Гуляев, А.И. Ипатов, М.И. Кременецкий, С.И. Мельников, Е.Е. Михайленко // Нефтяное хозяйство. 2012. - № 12. - С. 36-39.

4. Гуляев Д.Н., Лазуткин Д.М., Морозовский Н.С. Контроль разработки низкопроницаемых терригенных коллекторов по данным гидродинамических исследований скважин // Актуальные проблемы развития нефтегазового комплекса России: Сборник трудов XII Всероссийской научно-технической конференции. - М., 12-14 февраля 2018. - С. 82-91.

5. Пономарева И.Н. К обработке кривых восстановления давления низкопродуктивных скважин // Нефтяное хозяйство. 2010. - № 6. - C. 78-79.

6. Пономарева И.Н., Мартюшев Д.А., Ахметова М.И. Оценка оптимальной продолжительности проведения гидродинамических исследований низкопродуктивных скважин на примере Озерного месторождения // Нефтяное хозяйство. - 2016. № 1. - С. 60-63.

7. Савчик М.Б., Пономарева И.Н. Оценка состояния прискважинных зон при обработке недовосстановленных кривых восстановления давления // Вестник ПНИПУ. Геология. Нефтегазовое и горное дело. - 2012. - Т. 11. - № 2. - С. 77-82.

8. Ерофеев А.А., Пономарева И.Н., Мордвинов В.А. Обработка недовосстановленных кривых восстановления давления методами касательной и детерминированных моментов давления // Нефтяное хозяйство. - 2011. - № 10. - С. 25-27.

9. Гаврилов К.С., Сергеев В.Л. Адаптивная интерпретация нестационарных гидродинамических исследований скважин в системе «пласт-скважин» методом интегрированных моделей // Известия Томского политехнического университета. - 2012. T. 321. - № 5. - C. 72-75.

10. Сергеев В.Л., Ву К.Д. Адаптивная интерпретация результатов гидродинамических исследований с учетом влияния ствола скважины // Известия Томского политехнического университета. Инжиниринг георесурсов. - 2016. - Т. 327. - № 9. C. $70-77$.

11. Сергеев В.Л., Ву К.Д. Адаптивная интерпретация гидродинамических исследований горизонтальных скважин с идентификацией псевдорадиального потока / Известия Томского политехнического университета. Инжиниринг георесурсов. 2017. - T. 328. - № 10. - С. $67-73$.
12. Chaudhry A.U. Ch. 6: Fundamentals of pressure buildup analysis methods. Gas well Testing Handbook. - Houston, Texas, Advanced TWPSOM Petroleum Systems, 2003. -887 p.

13. Study on characteristics of well-test type curves for composite reservoir with sealing faults / Qiguo Liu, Heng Lu, Longxin Li, Aiting Mu // Petroleum. - 2018. - V. 4. - Iss. 3. - P. 309-317.

14. Hamidreza Hamdi Well-test response in stochastic permeable media // Journal of Petroleum Science and Engineering. - 2014. V. 119. - P. 169-184.

15. Разработка дизайна гидродинамического исследования в условиях карбонатного коллектора / А.Е. Давыдова, А.А. Щуренко, Н.М. Дадакин, А.Д. Шуталев, Б.Б. Квеско // Известия Томского политехнического университета. Инжиниринг георесурсов. - 2019. - Т. 330. - № 6. - С. 68-79.

16. Howard Crumpton. Well control completions and interventions. UK: Gulf Professional Publ., 2018. - 826 p.

17. Fei Wang, Shicheng Zhang. Pressure-buildup analysis method for a post-treatment evaluation of hydraulically fractured tight gas wells // Journal of natural gas science and engineering. - 2016. V. 35. - P. A. - P. 753-760.

18. Numerical well test model for caved carbonate reservoirs and its application in Tarim Basin, China / Yi-Zhao Wan, Yue-Wu Liu, Fang-Fang Chen, Neng-You Wu, Gao-Wei Hu // Journal of Petroleum Science and Engineering. - 2018. - V. 161. - P. 611-624.

19. Hedong Sun. Advanced production decline analysis and application. - UK: Gulf Professional Publishing, 2015. - 352 p.

20. Novel well test design for the evolution of complete well permeability and productivity for CSG wells in the Surat Basin / W. Bottomley, J. Schouten, E. McDonald, T. Cooney // Journal of natural gas science and engineering. - 2016. - V. 33. - P. 1002-1009.

21. A new numerical well testing approach: application to characterization of complex fault structures / B. Pouladi, M. Sharifi, M.R. Akbari, S.M. Hosseini-Nasab // Petroleum. - 2019. V. 5. - Iss. 3. - P. 295-302.

22. Hashmi G.M., Kabir C.S., Hasan A.R. Design and interpretation of transient tests at wells inception // Journal of petroleum science and engineering. - 2015. - V. 145. - P. 573-584.

23. Salam Al-Rbeawi. Deep insights to transient pressure behavior and stabilized productivity index of multilateral wells in laterally and spatially anisotropic reservoirs // Journal of natural gas science and engineering. - 2018. - V. 54. - P. 240-265.

24. Yi-Zhao Wan, Yue-Wu Liu, Fang-Fang Chen, Neng-You Wu, Gao-Wei Hu Numerical well test model for caved carbonate reservoirs and its application in Tarim Basin, China // Journal of Petroleum Science and Engineering. - 2018. - V. 161. - P. 611-624.

25. Мартюшев Д.А. Оценка эффективности проведения соляно-кислотных обработок по кривым восстановления давления // Газовая промышленность. - 2014. - № S (708). - С. 41-44.

Поступила 30.01.2019 2.

\section{Информация об авторах}

Мартюшев Д.А., кандидат технических наук, доцент кафедры нефтегазовых технологий Пермского национального исследовательского политехнического университета.

Слушкина А.Ю., инженер отдела проектирования и мониторинга разработки Чернушенской группы месторождений филиала 000 «ЛУКОЙЛ-Инжиниринг» «ПермНИПИнефть» в г. Перми. 
UDC 622.276

\title{
ASSESSMENT OF INFORMATIVE VALUE IN DETERMINATION OF RESERVOIR FILTRATION PARAMETERS BASED ON INTERPRETATION OF PRESSURE STABILIZATION CURVES
}

\author{
Dmitry A. Martyushev',
}

martyushevd@inbox.ru

\author{
Anna Yu. Slushkina², \\ slushkina2@yandex.ru \\ 1 Perm National Research Polytechnic University, \\ 29, Komsomolskiy aenue, Perm, 614990, Russia. \\ 2 Branch of LLC «LUKOIL-Engineering» «PermNIPIneft» in Perm, \\ 29, Sovetskoy Armii street, Perm, 614000, Russia.
}

The relevance of the research is caused by the fact that the main sources of information on filtration parameters of the remote zone of the formation are well tests at unsteady regimes with the recording of pressure or level recovery curves (pressure recovery curve or recovery curve). To determine the reliable parameters of the formation zone remote from the well, the duration of recording of the pressure recovery curve and the recovery curve should be long enough, which leads to losses in oil production. To determine the filtration characteristics of the formation, as well as reduce losses during hydrodynamic studies, it is possible to use the method of studying wells without stopping them - the method of stabilizing the pressure. However, now, the issue of analyzing and interpreting the results of measurements with the recording the pressure stabilization curves, which, like the pressure recovery curve, can give the required information about the formation, remains poorly understood.

The main aim of the study is the comparison of the informative value of reservoir parameters based on analysis and interpretation of pressure recovery curves, pressure stabilization curves and production analysis in the software product KAPPA Workstation v.5.20.01 (modules Saphir and Topaz).

Object: terrigenous reservoirs of deposits of Perm region.

Methods: modern software product of the $5^{\text {th }}$ generation KAPPA Workstation v.5.20.01 (modules Saphir and Topaz).

The results. The paper considers the particularly topical problem of well data interpretation from hydrodynamic studies in low-permeability reservoirs, because, due to low reservoir properties, well pressure is restored for long periods of time, and despite this, it is not always possible to achieve complete pressure recovery. In cases where the pressure reduction curves are not sufficiently recovered, it is not possible to reliably determine the filtration parameters of the formation, or it is possible to determine them evaluative. Pressure stabilization studies and production analysis, in their turn, in most cases have a longer duration than studies of the methods of the pressure recovery curve, and therefore, on the diagnostic chart, it is possible to clearly identify the radial filtration site and obtain reliable reservoir parameters. As a result, it can be concluded that when comparing the results obtained during the interpretation of the pressure recovery curves with the parameters obtained during the processing of the pressure stabilization curve and the extraction analysis, a high convergence is obtained, and the error is within $5 \%$.

\section{Key words:}

Well test, stabilization curve, pressure build-up curve, production analysis, low-permeability reservoirs,

formation filtration parameters, software product KAPPA.

\section{REFERENCES}

1. Ponomareva I.N., Milchakov S.V. Optimization of the pressure recovery period in oil producing wells during their exploration. Geology, geophysics and oil and gas field development, 2009, no. 9-10, pp. 36-39. In Rus.

2. Lin Jiaen, Yang Huizhu. Pressure buildup analysis using type curves for a well in a pressure-maintained system. Chinese jour nal of chemical engineering, 2007, vol. 15, Iss. 1, pp. 6-11.

3. Gulyaev D.N., Ipatov A.I., Kremenetsky M.I., Melnikov S.I., Mikhaylenko E.E. Management of development on the basis of long-term hydrodynamic monitoring on the example of the WestSalym field. Neftyanoe Khozyaystvo, 2012, no. 12, pp. 36-39. In Rus.

4. Gulyaev D.N., Lazutkin D.M., Morozovsky N.S. Kontrol razrabotki nizkopronitsaemykh terrigennykh kollektorov po dannym gidrodinamicheskikh issledovaniy skvazhin [Control of development of low-permeability terrigenous reservoirs according to the data of hydrodynamic studies of wells]. Aktualnye problemy razvitiya neftegazovogo kompleksa Rossii: Sbornik trudov XII Vserossiyskoy nauchno-tekhnicheskoj konferentsii [Proc. of the $12^{\text {th }}$ AllRussian Scientific and Technical Conference. Actual Problems of
Development of the Russian Oil and Gas Complex]. Moscow, 2018. pp. 82-91.

5. Ponomareva I.N. To the treatment of pressure recovery curves of low-productivity wells. Neftyanoe Khozyaystvo, 2010, no. 6, pp. 78-79. In Rus.

6. Ponomareva I.N., Martyushev D.A., Akhmetova M.I. Evaluation of the optimal duration of hydrodynamic studies of low-productive wells on the example of the Ozernoe deposit. Neftyanoe Khozyaystvo, 2016, no. 1, pp. 60-63. In Rus.

7. Savchik M.B., Ponomareva I.N. Evaluation of the condition of the wellbore zones during the treatment of unreduced pressure recovery curves. Vestnik PNRPU. Geology. Oil and gas and mining, 2012, vol. 11, no. 2, pp. 77-82. In Rus.

8. Erofeev A.A., Ponomareva I.N., Mordvinov V.A. Treatment of under reduced pressure recovery curves by the methods of tangential and deterministic pressure moments. Neftyanoe Khozyaystvo, 2011, no. 10, pp. 25-27. In Rus.

9. Gavrilov K.S., Sergeev V.L. Adaptive interpretation of non-stationary hydrodynamic well studies in the system of «wells» using the integrated model's method. Bulletin of the Tomsk Polytechnic University, 2012, vol. 321, no. 5, pp. 72-75. In Rus. 
10. Sergeev V.L., Wu K.D. Adaptive interpretation of the results of hydrodynamic studies taking into account the influence of the wellbore. Bulletin of the Tomsk Polytechnic University. Geo Assets Engineering, 2016, vol. 327, no. 9, pp. 70-77. In Rus.

11. Sergeev V.L., Wu K.D. Adaptive interpretation of hydrodynamic studies of horizontal wells with the identification of a pseudo-radial flow. Bulletin of the Tomsk Polytechnic University. Geo Assets Engineering, 2017, vol. 328, no. 10, pp. 67-73. In Rus.

12. Chaudhry A.U. Ch. 6: Fundamentals of pressure buildup analysis methods. Gas well Testing Handbook. Houston, Texas, Advanced TWPSOM Petroleum Systems, 2003. 887 p.

13. Qiguo Liu, Heng Lu, Longxin Li, Aiting Mu. Study on characteristics of well-test type curves for composite reservoir with sealing faults. Petroleum, 2018, vol. 4, Iss. 3, pp. 309-317.

14. Hamidreza Hamdi. Well-test response in stochastic permeable media. Journal of Petroleum Science and Engineering, 2014, vol. 119, pp. 169-184.

15. Davydova A.E., Shchurenko A.A., Dadakin N.M., Shutalev A.D., Kvesko B.B. Development of a design for hydrodynamic research in a carbonate reservoir. Bulletin of the Tomsk Polytechnic University. Geo Assets Engineering, 2019, vol. 330, no. 6, pp. 68-79. In Rus.

16. Howard Crumpton. Well control completions and interventions. UK, Gulf Professional Publ., 2018. 826 p.

17. Fei Wang, Shicheng Zhang. Pressure-buildup analysis method for a post-treatment evaluation of hydraulically fractured tight gas wells. Journal of natural gas science and engineering, 2016, vol. 35, P. A, pp. 753-760.

18. Yi-Zhao Wan, Yue-Wu Liu, Fang-Fang Chen, Neng-You Wu, Gao-Wei Hu. Numerical well test model for caved carbonate res- ervoirs and its application in Tarim Basin, China. Journal of Petroleum Science and Engineering, 2018, vol. 161, pp. 611-624.

19. Hedong Sun. Advanced production decline analysis and application. UK, Gulf Professional Publishing, 2015. 352 p.

20. Bottomley W., Schouten J., McDonald E., Cooney T. Novel well test design for the evolution of complete well permeability and productivity for CSG wells in the Surat Basin. Journal of natural gas science and engineering, 2016, vol. 33, pp. 1002-1009.

21. Pouladi B., Sharifi M., Akbari M.R., Hosseini-Nasab S.M. A new numerical well testing approach: application to characterization of complex fault structures. Petroleum, 2019. vol. 5, Iss. 3, pp. 295-302.

22. Hashmi G.M., Kabir C.S., Hasan A.R. Design and interpretation of transient tests at wells inception. Journal of petroleum science and engineering, 2015, vol. 145, pp. 573-584.

23. Salam Al-Rbeawi. Deep insights to transient pressure behavior and stabilized productivity index of multilateral wells in laterally and spatially anisotropic reservoirs. Journal of natural gas science and engineering, 2018, vol. 54, pp. 240-265.

24. Yi-Zhao Wan, Yue-Wu Liu, Fang-Fang Chen, Neng-You Wu, Gao-Wei Hu Numerical well test model for caved carbonate reservoirs and its application in Tarim Basin, China. Journal of Petroleum Science and Engineering, 2018, vol. 161, pp. 611-624.

25. Martyushev D.A. Evaluation of the effectiveness of hydrochloric acid treatments according to pressure recovery curves. Gas industry, 2014, no. S (708), pp. 41-44. In Rus.

Received: 30 January 2019.

\section{Information about the authors}

Dmitry A. Martyushev, Cand. Sc., assistant professor, Perm National Research Polytechnic University.

Anna Yu. Slushkina, engineer, branch of LLC «LUKOIL-Engineering» «PermNIPIneft» in Perm. 